\title{
L'habit à deux envers. Cupidité d'un lieutenant général et dignité de l'office royal au début du $\mathrm{XVII}{ }^{\mathrm{e}}$ siècle
}

\section{Thomas Lüttenberg}

\section{(2) OpenEdition}

Journals

\section{Édition électronique}

URL : http://journals.openedition.org/ccrh/2202

DOI : $10.4000 /$ ccrh.2202

ISSN : $1760-7906$

Éditeur

Centre de recherches historiques - EHESS

\section{Édition imprimée}

Date de publication : 20 octobre 1999

ISSN : 0990-9141

\section{Référence électronique}

Thomas Lüttenberg, «L'habit à deux envers. Cupidité d'un lieutenant général et dignité de l'office royal au début du xvII siècle », Les Cahiers du Centre de Recherches Historiques [En ligne], 23 | 1999, mis en ligne le 17 janvier 2009, consulté le 10 décembre 2020. URL : http://journals.openedition.org/ccrh/ 2202 ; DOl : https://doi.org/10.4000/ccrh.2202

Ce document a été généré automatiquement le 10 décembre 2020.

Article L.111-1 du Code de la propriété intellectuelle. 


\title{
L'habit à deux envers. Cupidité d'un lieutenant général et dignité de l'office royal au début du XVII ${ }^{\mathrm{e}}$ siècle
}

\author{
Thomas Lüttenberg
}

1 L'idée d'une administration royale juste et efficace, telle que Claude de Seyssel l'a exprimée en 1519, est un des sujets importants du débat politique au $\mathrm{XVI}^{\mathrm{e}}$ siècle : cette administration est fondée sur « tant de bonnes lois, coutumes et ordonnances », écrit-il ; « il ne faut que bons ministres pour les bien garder et observer, et qui n'aient égard sinon au bien public $»^{1}$. On sait que le lien entre vertu et utilité, fondamental dans la théorie politique du Moyen Âge tardif, fut systématiquement rompu par Machiavel dont les écrits suscitèrent, surtout vers la fin du XVI $\mathrm{I}^{\mathrm{e}}$ siècle, de violentes réactions partout en Europe ${ }^{2}$. En France, la discussion des œuvres de Machiavel vint se mêler au conflit confessionnel. Le débat politico-religieux trouva une expression particulière dans le domaine de l'administration de la justice, et ce furent les juges eux-mêmes qui créèrent une doctrine moralisée de leurs devoirs. Dans la pratique toutefois, l'existence d'une déontologie plus rigide ne bénéficia pas forcément aux institutions de la justice royale, au contraire, les juges continuèrent à officier en fonction de leurs intérêts propres plutôt qu'en vue du bien public, et cela avant tout à cause de la vénalité des offices, qui introduisait les intérêts personnels des officiers - certains diront: leurs vices - dans le domaine de la justice. Dans la perception de l'écart entre doctrine et pratique, une image semble avoir joué un rôle particulier. Il s'agit de la métaphore de la robe du magistrat, qui unissait dignité de l'office et imperfections des officiers. Le présent travail a pour objet de développer quelques réflexions sur le rapport entre la cupidité des juges, la déontologie de la justice et la métaphore de la robe du magistrat. Commençons par un exemple tiré du milieu des officiers « moyens » du siège présidial de la bonne ville de Bourges, capitale du Berry. 


\section{Les épices du lieutenant général au siège présidial de Bourges}

2 Pierre Biet avait accédé à la charge de lieutenant général au siège présidial de Bourges en $1582^{3}$. Allaient lui succéder sans interruption, sur le poste le plus important de l'administration royale en la capitale du Berry, trois autres membres de la famille Biet, formant ainsi une dynastie qui occupa la lieutenance générale de la capitale du Berry pendant plus de cent ans, jusqu'au début du xvIII siècle. L'histoire de cette famille illustre parfaitement la théorie selon laquelle l'office est au cœur de l'ascension sociale en province, ascension qui commença, dans le cas de la famille Biet, par le père de Pierre, venu à Bourges vers le milieu du XVI ${ }^{e}$ siècle afin d'y poursuivre des études de droit. Cet ancêtre acheta ensuite des offices «moyens » de justice, il fut notamment conseiller au présidial et lieutenant particulier. Les descendants de Pierre Biet réussirent leur intégration dans la noblesse régionale, intégration achevée au plus tard en 1667 par le mariage d'une petite-fille de Pierre Biet avec un descendant d'une des plus anciennes familles du Berry, les Ménil-Simon ${ }^{4}$. Au début de cette ascension, l'exercice de la charge de lieutenant général par Pierre Biet fut exceptionnellement long: en effet, il joua un rôle important dans l'administration de la justice en Berry pendant plus de quarante ans, de 1582 à 1624 .

3 Pendant ces longues années de service, son comportement professionnel ne fut pas toujours irréprochable. D'une manière générale, les abus de pouvoir ne sont pas faciles à déceler, leur nature illégale portant leurs auteurs à les dissimuler. Que les méfaits de Pierre Biet aient été découverts et portés au grand jour est dû à une plainte déposée au parlement de Paris par deux de ses collègues au présidial de Bourges, les conseillers Pierre Bidault et Gabriel Manceron. Au cours de cette action en justice, ils constituèrent un factum pour exposer leurs arguments ${ }^{5}$.

4 Ce texte, long de près de cinquante pages, fut très probablement rédigé en 1614. Il présente une multitude de cas précis dans lesquels le lieutenant général aurait commis des infractions aux lois, notamment autour de la prise d'épices. Afin de l'empêcher de continuer dans cette voie, les deux conseillers demandèrent au parlement

[...] suspension, interdiction de sa charge durant le cours et instruction du proces,

décret d'adjournement personel, prise de corps, ou autres salutaires remèdes... $»^{6}$.

Les infractions dont s'était rendu coupable le lieutenant général avaient pris des formes diverses, mais leur but avait toujours été l'enrichissement personnel :

[...] ledit Biet ne laisse passer aucune occasion de remplir sa bourse?

L'éventail des délits comporte plusieurs sortes de taxation illégale, dont la prise d'épices sans motif valable ou en infraction aux règlements. Le lieutenant général avait ainsi taxé des actes judiciaires qui n'y étaient normalement pas sujets, comme la publication de testaments ou de contrats de mariage. Dans un autre cas, daté de 1600, on voit que Biet s'était mis d'accord avec le prévôt de la maréchaussée afin de pouvoir siéger aux procès de la maréchaussée et d'y prendre des épices comme les autres juges. Cela n'avait sans doute pas posé de difficultés : on imagine que, les épices de la maréchaussée se taxant par tête à Bourges, les autres juges ne durent pas offrir une quelconque résistance à cette action, la part de Biet ne diminuant pas la leur... Le lieutenant général avait enfin simplement inventé des épices, comme le soi-disant « droit de tapy » que Biet prenait 
[...] pour la distribution et numération qui se fait en son hostel des deniers procédant des décrets. Il se fait payer grandes sommes par chacun créancier qui reçoit ${ }^{8}$.

Ainsi se poursuit la liste des nombreux abus, commis, aux dires des deux adversaires du lieutenant général, sans gêne aucune et avec une grande assurance.

Comment Pierre Biet a-t-il réussi, pendant une période aussi longue, à contourner les préceptes du bon service royal ? Ses abus, les adversaires le soulignent dans leur factum, ont été rendus possibles grâce au large soutien apporté par sa famille et par sa clientèle. Bidault et Manceron insistent entre autres sur le fait que l'abus de pouvoir continu dont le lieutenant général s'est rendu coupable reposait sur une aide provenant de l'intérieur comme de l'extérieur du présidial, grâce aux nombreux membres de sa famille installés peu à peu dans l'administration de la justice, avant tout au sein du siège présidial, mais aussi dans d'autres institutions, comme la prévôté de Bourges et la maréchaussée du Berry. Rien qu'au sein du présidial, les deux conseillers ne dénoncent pas moins de sept parents du lieutenant général, chiffre qu'on peut vérifier en établissant la prosopographie du siège et qui s'est révélé parfaitement correct ${ }^{9}$. En outre, le prévôt de la maréchaussée qui avait couvert les tractations de Biet quand celui-ci avait demandé de siéger à ses côtés aux procès de la maréchaussée, était le gendre de Biet, lien qui rendait un refus impossible. Biet s'était aussi acquis des officiers au-delà de sa parentèle, par des pressions voire par des pots-de-vin, deux autres délits qui ternissent encore davantage l'image de l'officier en question. Nous sommes donc en présence d'un cas d'abus de pouvoir commis par un officier exerçant une fonction importante de l'administration royale en province, officier qui était en même temps à la tête d'un clan familial et clientélaire qui le soutenait, bon gré mal gré, dans ses desseins.

Quelle fut la réaction de l'institution de tutelle, le parlement de Paris ? Il faut croire qu'elle fut nulle. L'issue du procès est inconnue, mais nous savons que Biet n'a pas été destitué de son office. Il le résigna tranquillement, dix ans plus tard, entre les mains de son fils aîné ${ }^{10}$. Absence de contrôle efficace, existence d'un réseau familial au sein du présidial, telles furent les conditions dans lesquelles les abus d'épices du lieutenant général s'accrurent - et sa fortune avec. Mais revenons au factum de Bidault et Manceron, car la liste de leurs griefs n'est pas épuisée. À l'extrême fin du texte, une fois l'énumération des abus terminée, ils s'intéressent à la vie privée du lieutenant général, qualifiée de « lubrique et dissolue » :

Bref, puis qu'il faut faire cognoistre la vérité, ledict Maistre Pierre Biet est reprehensible, non seulement dans sa charge, mais en les mœurs, ayant... despuis douze ou quinze ans en ça tousiours mené une vie dissolue, et entretenu publiquement des concubines, dont il y a enfans, au veu sceu d'un chacun, les allans visiter ordinairement, et de plain jour avec ses habits de Palais, au grand scandale d'un chacun... ${ }^{11}$.

7 Il est évident que Bidault et Manceron voulaient donner plus de poids à leur plainte, en reprochant au lieutenant général non seulement des fautes professionnelles mais également son inaptitude morale au service de justice. Vêtir la robe dans une situation aussi compromettante portait en effet atteinte à la dignité du service royal. En accusant Biet d'aller voir ses concubines de plein jour, situation qui ne sied pas à qui détient une part de la puissance publique, ses adversaires dépeignent-peu importe que leur accusation soit vraie ou fausse - l'incurie absolue de Biet face aux exigences de dignité et de suffisance. L'image est couronnée par le fait que le lieutenant ne juge même pas nécessaire de se défaire de sa robe, son habit de palais, insigne de la dignité de son office. 
Deux points de cet ultime passage du factum sont assez curieux. Premièrement, pourquoi ses ennemis évoquent-ils ainsi la vie privée et les qualités morales du lieutenant général, quand il s'agit apparemment avant tout (pendant quarante pages) de le juger sur ses qualités professionnelles ? Et ensuite, quel rôle la métaphore de l'habit de palais joue-t-elle dans la disqualification du juge? La réponse à la première question est liée à la déontologie de la justice royale, telle qu'elle a été mise en place dans la seconde moitié du $\mathrm{XVI}^{\mathrm{e}}$ siècle. L'autre question - question de discours - y est liée, mais elle relève aussi d'autres types d'explication.

\section{La déontologie de la justice royale}

8 Ce qui fait un bon juge, Pierre Biet l'a peut-être lu, trois ans après le factum, dans les Treize Livres des Parlemens de France, publié à Bordeaux en 1617. L'auteur, Bernard de La RocheFlavin (1552-1627), président de la chambre des requêtes du parlement de Toulouse, y présente, en de longs chapitres, une doctrine détaillée du comportement des magistrats. La Roche-Flavin n'en est pas l'inventeur : il ne fait que systématiser les règles développées au cours de la seconde moitié du XvI ${ }^{e}$ siècle dans les milieux parlementaires. Relevant du lent processus de la « confessionalisation » catholique ${ }^{12}$ et du courant hostile aux idées de Machiavel, la doctrine du "parfait magistrat catholique " visait une moralisation des juges dont la genèse et les buts lointains - le renforcement de la cohésion sociale de la magistrature et sa séparation des autres groupes urbains par la création de l'image d'une supériorité irréprochable - ont été magistralement décrits par Colin Kaiser ${ }^{13}$. En 1617, le livre de La Roche-Flavin présente la panoplie détaillée de ces devoirs ${ }^{14}$. Le bon magistrat doit non seulement être un professionnel qui exerce son office conformément aux ordonnances royales et aux règles de sa corporation, mais il lui faut aussi être un homme pieux, entendant régulièrement la messe, un guide sur les chemins de la foi, montrant au peuple un bon exemple de piété et dévotion ${ }^{15}$. Il doit agir en toutes circonstances avec modération, ce qui implique le renoncement à un style de vie luxueux. S'y ajoute finalement le devoir d'intégrité, que La Roche-Flavin appelle " pureté des mains $»^{16}, c^{\prime}$ està-dire le refus de tout don ou présent offert par les parties et généralement de toute action déshonorante. En conséquence, le bon juge ne doit pas se montrer dans des situations moralement compromettantes, et particulièrement dans les lieux publics où l'on joue, parce que le risque d'y fréquenter des gens peu convenables est trop grand ${ }^{17}$.

9 On est évidemment tenté de croire que c'est l'image très moralisée du juge parfait que les deux adversaires du lieutenant général de Bourges avaient à l'esprit lorsqu'ils dénoncèrent la vie dissolue de Biet : entretenir des concubines et les visiter en plein jour était bien plus grave que participer à un jeu de hasard. En même temps, la référence au code déontologique que les deux conseillers placent à la fin de leur factum rappelle ses fautes professionnelles, la prise d'épices exagérée, qui, elle aussi, est susceptible de subir un jugement moral. Il est clair que dans le cas de Biet, on ne peut point parler de pureté des mains. La dénonciation de son comportement professionnel comme de sa vie privée mettait le lieutenant général sous une pression énorme, car les règles étaient valables pour tous les juges, souverains ou non. Bien sûr, le code déontologique concernait au premier chef les membres des parlements, dont la position à la tête de la justice faisait de leur comportement une véritable question politique ${ }^{18}$. Néanmoins, ce code était en principe destiné à tous les magistrats, donc également aux juges de province, «moyens » ou inférieurs ${ }^{19}$. Partout, au sein des cours souveraines comme au niveau des justices 
inférieures, à Paris comme en province, le "parfait magistrat catholique " aurait dû exercer son office avec la rigueur exemplaire dont La Roche-Flavin exposait les principes - et contre lesquels le lieutenant général du présidial de Bourges, selon ses adversaires, avait péché avec une insouciance choquante, "au grand scandale d'un chacun ".

Le scandale se nourrissait aussi du fait que le lieutenant avait agi publiquement, soulignant de cette façon le peu de prix qu'il attachait à son image. Or, la nouvelle doctrine faisait de l'apparence des juges et de leur image publique un point capital. L'enjeu politique de la «confessionalisation » catholique exigeait une extériorisation des valeurs décrites ci-dessus. Il fallait que la foi catholique et l'intégrité, la modération et la gravité des juges soient visibles. Afin de donner de la crédibilité à la réforme, il ne suffisait pas d'être un bon juge, il fallait aussi le paraître, pour donner l'exemple à la population. Selon ces critères, Pierre Biet avait doublement mal agi. Non seulement il ne se souciait aucunement de son image, mais son comportement ne pouvait non plus servir d'exemple, ce que soulignent les deux conseillers, prétendant que le scandale avait particulièrement agi sur

[...] la jeunesse et escholiers qui sont en l'université dudit Bourges, lesquels par ce mauvais exemple prennent occasion de se licentier et abandonner aux vices et desbauches, et à une vie lubrique et dissolue ${ }^{20}$.

Il n'est guère étonnant de voir que la nouvelle déontologie, destinée à extérioriser les qualités des juges afin de démontrer leur supériorité, ait réservé une place de choix aux questions vestimentaires. Parce que l'apparence d'un magistrat en public pouvait avoir des conséquences pour l'image du groupe tout entier, il fallait éviter la « dissolution » et $l^{\prime}$ « indécence » des vêtements, un argument qui retint l'attention des juges dès le départ ${ }^{21}$ . Selon La Roche-Flavin, il est

[...] bienséant que les conseillers souverains des parlements soient distingués par différence d'habits et vêtements de l'autre peuple ${ }^{22}$.

La discussion autour de la nécessaire correspondance entre les qualités intérieures des juges et leur apparence vestimentaire touchait en fait au problème fondamental de ce temps de «troubles » que fut la période des guerres de religion, c'est-à-dire le sentiment général de la dissolution de la cohésion sociale. Le sentiment de désorientation aurait eu pour conséquence une dissolution des mœurs, en particulier en ce qui concerne les signes extérieurs d'identité sociale, le vêtement. En s'appuyant sur la très riche tradition du Moyen Âge des sermons ad status, on se plaignait de l'impossibilité générale de pouvoir opérer la différence, dans la rue, entre un bourgeois et un noble :

En France ce n'est que confusion. On ne peut discerner le serviteur du maistre, un homme de bonne et honneste famille d'un artisan, un justicier ou magistrat d'un sergent ou d'un procureur [...] Ce seroit un reiglement fort utile d'y pourveoir pour le passé, et pour l'advenir obliger, comme cela se voyoit du temps des roys predecesseurs, que la différence des conditions se peust recognoistre par les habits ${ }^{23}$.

11 C'est en réponse à ce genre de plainte que les efforts réformateurs de Charles IX et surtout d'Henri III apportèrent une intensification de la législation somptuaire, constituée d'ordonnances visant à réprimer le luxe ostentatoire de manière générale et la confusion vestimentaire des états en particulier. À partir de 1561, ces textes ont détaillé une classification sociale, destinée à prescrire son apparence à chaque groupe social, et ce en vue de rétablir un ordre manifestement perturbé24. Sur fond de cette discussion tournant autour de la visibilité des qualités de chacun par son vêtement - » ce n'est pas assez d'être bon et grave, il faut que nous paraissions tels » dira La Roche-Flavin au nom 
des juges ${ }^{25}$ - la robe longue du magistrat gagnait une importance primordiale en tant que métaphore des qualités de ce dernier. Mais la spécificité de cette métaphore menait en même temps à son retournement, et elle fut tout autant utilisée pour dénoncer les défauts de la justice. Comment l'image de l'habit du juge glissa-t-elle d'une démonstration de son comportement idéal vers une représentation des ambiguïtés ouvertes entre l'aspiration au modèle parfait et une réalité souvent décevante? La réponse à cette question permettra de mieux situer l'image de l'habit de palais utilisée à l'encontre du lieutenant général de Bourges.

\section{La robe du magistrat}

En principe, la robe du magistrat représente sa dignité. C'est un signe du pouvoir conféré par le roi. Pour illustrer cette investiture, la robe du magistrat est souvent identifiée à celle du roi :

Il est certain que l'habit donné par les Roys à Messieurs de Parlement (i'entens de Messieurs les Presidens) estoit le vray habit dont estoient vestues leurs Majestez. Cet habit leur a esté donné, a fin qu'estant habillez comme le Roy, on creust que les arrests qu'ils donnoient estoient arrests de Roy, et eussent pareille authorité que ceux prononcez pour le Roy ${ }^{26}$.

13 L'habit des magistrats - au moins celui des présidents du parlement - était composé de trois éléments : la simarre, une sorte de soutane de soie noire, la robe, longue et à larges manches de velours, de couleur écarlate pour les cérémonies et noire pour l'usage quotidien, et le manteau de pourpre bordé d'hermine ${ }^{27}$. Jouant ainsi sur les ressemblances de couleur (écarlate) et de matière (hermine, velours, soie), les vêtements des juges étaient le signe patent de leur identification à la personne royale. Cette mise en valeur et des juges et de leur robe par une participation matérielle à la majesté du roi correspond parfaitement au nouvel idéal du magistrat, idéal destiné à séparer les magistrats du reste de la population.

Malheureusement pour les juges soucieux de leur image, la réalité ne satisfaisant souvent pas aux exigences de la théorie. Malgré un profil professionnel plus rigoureux, la vénalité des offices continuait à autoriser l'accès aux offices de justice pratiquement sans égard pour les qualités personnelles des officiers. De fait, il se pouvait que la robe fût portée par des hommes dont les qualités morales et professionnelles ne correspondaient en rien à la splendeur de leur apparence. La robe écarlate dissimulait souvent des défauts à l'opposé précis des qualités requises, comme le dénonçait en 1621 Thomas Sonnet, sieur de Courval (1577-1627), auteur satirique auquel on doit une Satyre Ménippée violemment misogyne, ainsi que plusieurs autres satires, dont un ouvrage intitulé «Contre la corruptelle et malversation des pervers officiers de judicature » :

[...] Ces fils de gros marchans et riches usuriers,

Qui a force d'argent se sont faicts officiers,

Conseillers, presidens, voulans tenir les sièges

Et le rang de lyons dans ces divins colleges

Consacrez à Themis, ils n'en ont seulement

Que la robbe et la peau : car sous ce vestement

Sous ce roquet fourré et robbe d'escarlatte

Un gros asne est caché qui se mire et se flatte $[. . .]^{28}$.

Sonnet critique avant tout le profond clivage qu'il perçoit entre la dignité de l'office, symbolisée par la «robbe d'escarlatte», et l'insuffisance des officiers en exercice. 
Comment les ânes ont-ils pu accéder au rang des lions? Simplement en revêtant leur robe, bien sûr, mais cela ne les qualifie pas automatiquement, loin s'en faut.

L'habit ne fait pas le moine, et Furetière explique ce que le proverbe veut dire :

[...] ce n'est pas assez de marquer à l'extérieur sa profession, il faut encore en observer la régularitée 29 .

Sonnet, qui était docteur en médecine, avait en 1610, publié une autre satire, «Contre les charlatans et pseudomédecins $»^{30}$, dans laquelle il dénonce justement l'absence de régularité dans cette profession. La règle à laquelle manquaient, selon lui, les juges, était celle de la dignité, et c'est la vénalité des offices qui permettait à l'argent de l'emporter sur la probité. Le contraste choquant entre la dignité conférée par l'office et le fait que n'importe qui pouvait faire l'acquisition d'un office pourvu qu'il pût en débourser le prix, est le point d'orgue de toutes les critiques de ce système. Parce que le roi vend les offices au plus offrant au lieu de les conférer au plus digne, la porte de la justice royale est largement ouverte aux vices. D'ailleurs, la question n'est pas seulement posée au niveau d'une critique générale, moralisatrice, elle vise un vice particulier, la cupidité des officiers de justice ${ }^{31}$. L'investissement souvent considérable auquel devaient consentir les «fils de gros marchans » afin d'accéder aux honneurs de la justice royale exigeait un rendement au moins raisonnable, ce qui signifie que les officiers devaient compenser cet investissement de départ d'une manière ou d'une autre, ne serait-ce qu'auprès des justiciables. Sonnet, qui se souciait avant tout de l'abus de pouvoir commis quotidiennement par les officiers de justice - ce n'est pas pour rien qu'il écrit « contre la corruptelle et malversation »- explique ce mécanisme :

Chacun veut à son tour avoir part à la proye, et tasche, en butinant, en détail rembourser, ce qui luy a fallu tout en gros desbourser... ${ }^{32}$.

Ainsi sa description du décalage entre la dignité de l'office et les insuffisances des officiers ne vise pas seulement la vanité de ces derniers, mais particulièrement leur cupidité, défaut principal engendrant corruption et malversation. Ce sont des hommes comme le lieutenant général Pierre Biet, avides de s'enrichir grâce à leur office, que la critique de Sonnet met sur la sellette. Une cupidité sans limites fut le défaut de beaucoup d'officiers, et il est fort possible que les juges des juridictions moyennes et inférieures (présidiaux, bailliages, sénéchaussées et prévôtés) s'en soient rendus particulièrement coupables, comme l'insinuait l'avocat général au parlement de Dijon à la fin du XVII siècle. Dans ses harangues, prononcées lors de sessions mercuriales de sa cour, il fustigeait l'avarice des juges inférieurs comme étant leur principal défaut :

Il semble que ce soit le seul but de toutes leurs actions et qu'ils ne rendent la justice qu'autant qu'ils en retirent du profit... leur avarice est crûe jusqu'à cet excez qu'ils ne gardent plus aucune mesure dans les taxes d'épices qu'ils se font ${ }^{33}$.

18 Thomas Sonnet, en tant qu'auteur satirique, ne s'arrête d'ailleurs pas à une simple critique directe de la cupidité officière. Le médecin normand la dote, quelques vers plus loin, d'une autre image, identifiant les officiers à l'ambre que l'on croit trouver au bord de la mer et dont on s'aperçoit en se rapprochant qu'il ne s'agit que de l'écume :

[...] Gens qu'on peut comparer à la grisastre escume

Que Thetis en ses bords rejette de coustume,

Qui nous semble de loin ambre gris proprement,

Et, touchée à la main, n'est qu'un vil excrement.

Ces asnes d'officiers sont de mesme nature :

Car, à leur voir de loin d'officiers la vesture,

L'escarlatte pourprin, marque de majesté,

Une bonne façon pleine de gravité, 
On diroit que ce sont senateurs de Venise ;

Mais, à taster de près si belle marchandise,

Ce n'est rien qu'excrement remply de vanité $[. . .]^{34}$.

19 Dans ce passage, l'image de l'écume mène directement à celle de l'excrément. Il est évident qu'afin de souligner la gravité du problème des abus, l'auteur creuse encore davantage le fossé entre les métaphores de la dignité du service et l'insuffisance des candidats, les principaux attributs de la première comme de ces derniers étant accentués dans deux directions opposées. D'un côté, l'éclat de la robe écarlate est rehaussé par la référence expressis verbis à la majesté, de l'autre, les officiers sont rabaissés par une identification sans ambiguïté aux excréments. On pourrait bien sûr trouver à Sonnet une sensibilité psychanalytique avant la lettre, mais l'image selon laquelle la cupidité est sale était en fait très répandue ${ }^{35}$. L'accouplement des deux termes chez Sonnet n'a d'ailleurs rien de neuf. Critiquer la cupidité en la comparant aux excréments est une tradition qui remonte au début $d u x{ }^{e}$ siècle, qui vit l'apparition du thème iconographique des hommes et des singes déféquant de l'or ${ }^{36}$. On retrouve l'image, mais de manière inversée, dans l'Utopia de Thomas More (1516), où il est question du "magnifique mespris de lor » par lequel s'illustrent les habitants de l'île. Voici les termes mêmes du passage dans la traduction française qui parut en 1550 à Paris :

Certes on ne scauroit croire comme les Utopiens ont lor et largent à petite reputation ... leurs potz à uriner et autres vesseaux qui servent à choses immundes sont dor et dargent, pareillement les chaînes et gros fers, dequoy sont detenuz et liez leurs criminelz $[. . .]^{37}$.

More rapproche l'excrément de la cupidité dans sa forme négative, la "petite réputation » de l'or. Cette vision reflète une situation réelle, où bien entendu peu de personnes ne sont indifférentes aux attraits de l'or, situation moralement condamnable et qui selon l'auteur devrait être inversée. Quoi qu'il en soit, l'équivalence entre or et choses immondes dont Sonnet se sert afin de dénoncer la cupidité des officiers est une image forte, qui autorise un contraste expressif avec les valeurs de probité, d'intégrité et de gravité réunies dans la métaphore de la robe. La combinaison métaphorique de la robe et de l'excrément, deux éléments fortement opposés et cependant proches, rappelle Pantagruel dénonçant le travail des glossateurs du droit romain :

Et disoit aulcunesfois que les livres des loix luy sembloyent une belle robbe d'or, triumphante et precieuse à merveilles, qui feust brodée de merde, car disoit il, au monde n'y a livres, tant beaulx, tant aornés, tant elegans, comme sont les textes des Pandectes, mais la brodure d'iceulx, c'est assavoir la glose de Accurse, est tant salle, tant infame, et punaise, que ce n'est que ordure et villenie ${ }^{38}$.

21 Il y a un parallèle frappant entre les deux images, celle de Rabelais et celle de Sonnet. De la même manière que la robe d'or du droit romain a été souillée par les glossateurs, la robe du magistrat est souillée par les défauts des officiers indignes. Mais tandis que chez Rabelais, la robe d'or a été ouvertement et visiblement couverte d'excréments, chez Sonnet, la robe du juge cache les vices, sans que cela puisse éviter la souillure. Il est inévitable que l'habit du magistrat entre en contact avec les excréments, bien plus, les vices des officiers ne peuvent que souiller - métaphoriquement - la robe écarlate, même si seul l'envers de la robe est sali. Le potentiel de cette métaphore - le dessous de la robe souillé par la cupidité de son porteur - donne un sens plus riche à la mention de l'habit de palais que les deux conseillers berruyers font dans leur factum. Ils dénoncent explicitement la souillure de l'habit par la vie dissolue que mène le lieutenant général, mais ils laissent entendre que la robe du juge est bien plus tachée par sa cupidité, vu la 
grande importance qu'ils accordent dans leur plainte à la prise d'épices démesurée. L' habit de palais du lieutenant général fut donc doublement souillé, car ni son attitude professionnelle ni son comportement privé ne correspondaient aux exigences de la déontologie des juges.

\section{L'habit à deux envers}

Une chose reste tout de même évidente chez les deux conseillers, comme chez Rabelais et Sonnet, c'est que, finalement, la distinction entre le bien et le mal demeure claire. Bidault et Manceron visent l'homme, pas l'office. Rabelais, quant à lui, marque parfaitement la différence entre les valeurs éternelles du droit romain et les griffonnages des glossae, et ce que décrit Sonnet est un défaut propre aux officiers, pas une corruption de l'office. Dans les trois textes, la souillure ne fait pas partie de la robe. Le potentiel de l'image de la robe souillée ne se limite cependant pas à une critique des officiers indignes, comme on peut l'observer dans le développement d'un discours politique qui fait précisément usage de cette image.

Cela ressort clairement d'un petit ouvrage datant de la même époque que le factum contre Biet et la satire de Sonnet. Il s'agit d'un pamphlet anonyme, en vers, composé en réaction à une ordonnance somptuaire inconnue, et intitulé Pasquil ou Plaincte sur la Reformation des Habits. Il a la particularité de fusionner les deux sphères (la robe splendide et la souillure de son dessous) que Sonnet et les deux conseillers au présidial de Bourges séparaient soigneusement. Le pamphlet est certainement postérieur à l'avènement de Louis XIII, car il est question de "nostre Louys $»^{39}$. La datation manuscrite de l'exemplaire conservé à la Bibliothèque nationale porte l'année 1613, date qui doit être considéré comme terminus post quem. Le Pasquil anonyme pourrait donc bien se référer à l'ordonnance somptuaire de mars 1613 et faire partie du groupe de pamphlets rédigés autour des états généraux de $1614^{40}$. Entrent en scène sept groupes, qui représentent les états visés par l'ordonnance somptuaire, par suite de leur trop importante consommation d'étoffes de luxe. Les princes, les cardinaux, la haute noblesse et la noblesse de campagne, les membres du conseil royal, la justice et le tiers état, tous vêtus d'étoffes simples, parfois même grossières, défilent et se plaignent d'être réduits par la volonté du roi à un état vestimentaire trop modeste. Ainsi, les princes sont vêtus d'habits de treillis, une toile épaisse dont on faisait la garniture des corps de pourpoint pour les renforcer; la haute noblesse en bougran, une grosse toile utilisée pour doubler les vêtements et leur donner du tombant, et la noblesse de campagne en moquette, qui, selon Furetière, était une étoffe de laine dont « on fait les emmeublemens communs $»^{41}$. Le texte puise sa force comique dans l'exagération de la modestie imposée par l'ordonnance royale : il montre les groupes dans une situation pénible, à laquelle de surcroît ils ne peuvent échapper : la noblesse, habituée à la douceur et au scintillement du velours par exemple, ressent maintenant la rudesse d'une étoffe de rembourrage, le bougran.

Ce procédé n'est pas le seul moyen utilisé pour retenir l'attention du lecteur. Le choix des étoffes attribuées aux différents groupes respectifs n'est pas laissé au hasard, bien au contraire, il renvoie à leurs principaux défauts. Cela ressort plus clairement dans le cas des deux groupes désignés par leur fonction plutôt que par leur appartenance à l'un des trois états. Premiers à être évoqués, ces « Messieurs du Conseil » sont critiqués pour leur inconstance, qui fait que leurs décisions changent instantanément, au gré de leur humeur. L'habit approprié à leur défaut est donc de taffetas changeant : 
Messieurs du Conseil, de taffetas changeant :

Et nous qui sommes du Conseil,

L'on nous donne le nompareil

A ce taffetas de la Chine,

Mais nous pouvons en un moment

Faire que nostre habillement

Se change ainsi que nostre mine

Car nous pouvons, si nous voulons

Rompre tout ce que nous faisons ${ }^{42}$

La légèreté des hommes qui sont censés prendre les décisions les plus graves est exprimée par cette qualité de taffetas. Qu'en est-il pour la justice? Le principe - illustration du défaut par une étoffe - reste le même, mais le tissu a changé :
La justice, de serge à deux envers :
Faut-il que nos satins divers
Se tourne en serge en deux envers
A nous qui tenons la justice,
Hélas, ouy, nous le meritons
Et s'il n'y a de grands pardons
Le gibet est notre supplice
Car l'endroict de tous les procez
Nous le retournons à l'envers ${ }^{43}$.

Il est évident que le défaut le plus grave pour un juge est d'être injuste. L'injustice dénoncée ici tourne l'endroit des procès à l'envers. Le manque d'impartialité est un défaut si grave qu'il mériterait la mort, et il faut que les juges soient mus par de forts mobiles pour délaisser à ce point leurs devoirs. Quel intérêt peut les pousser à agir de la sorte ? Il nous semble que c'est justement leur intérêt propre, la cupidité que dénonçaient Sonnet et les critiques du système de la vénalité des offices, qui font que l'idéal d'un jugement équitable passe au second rang, au profit de la richesse de chaque magistrat. Dans la procession des états que le Pasquil déploie sous nos yeux, ce défaut trouve son illustration littéraire dans le port d'un drap simple, la serge à deux envers. Il s'agit d'une étoffe commune et légère, de laine, dont s'habillent les pauvres, toujours selon Furetière.

Les gens de justice, d'habitude vêtus de soie et de satin, méritent d'après le texte des habits simples, qui ont en plus la particularité d'être réversibles: on peut les porter à l'endroit comme à l'envers. Le choix de cette étoffe renvoie simplement, tout d'abord, à l'image des procès tournés de l'endroit à l'envers. Mais il n'est pas non plus sans rappeler les attributs complexes de la robe du magistrat, que nous avons exposés, ce rouge écarlate qui cache les vices-excréments des officiers. La nouvelle étoffe rend visible ce qui jusquelà était demeuré caché : en retournant la robe du juge, l'éclat disparaît et le dessous souillé voit la lumière du jour.

L'exagération métaphorique du Pasquil fait ressortir le vrai caractère de l'exercice de la justice. Ce caractère n'est pas uniquement vicieux, il se distingue plutôt par une ambiguité. L'exercice de l'office du magistrat se situe entre les deux pôles de la déontologie et de ses intérêts propres. La métaphore de l'habit à deux envers est donc une expression tout à fait juste de la condition équivoque du juge. Mais elle est encore plus efficace, puisqu'elle élargit la critique au service même de la justice.

La séparation nette établie par Sonnet entre robe et souillure permettait à chaque juge d'être en accord avec cette critique, puisqu'on pouvait toujours prétendre ne pas faire partie de la mauvaise tranche de la magistrature et qu'on pouvait ainsi faire reporter la responsabilité de la souillure sur les brebis galeuses; les deux conseillers, quant à eux, se 
servaient de cette distinction pour mieux dénoncer Pierre Biet. Mais l'image de la réversibilité de la serge intègre le vice de cupidité jusqu'alors cantonné à des individus au sein même du service royal ; elle ajoute une dimension politique à l'image de l'habit à deux envers. Dénoncer les défauts intrinsèques du système est une critique $\mathrm{ad} \mathrm{rem,} \mathrm{non} \mathrm{ad}$ personam. Parce que la pratique de la vénalité des offices incite les juges à donner libre cours à leur cupidité, ce ne sont pas seulement les mauvais officiers qui sont responsables de la situation, mais tout le système de l'administration de justice à charges vénales.

Comme l'envers d'un vêtement est inséparable de l'endroit, la cupidité encouragée par la vénalité des offices devient partie intégrante de l'administration publique. La déontologie de la justice royale, qui exige modération et intégrité, et la cupidité des officiers, qui est encouragée par la vénalité des charges, s'entrelacent comme la trame et la chaîne d'une étoffe : elles ne sont que les deux faces d'un même habit.

\section{NOTES}

1. Claude de Seyssel, La Monarchie de France et deux autres fragments politiques (1519), Jacques Poujol, éd., Paris, Librairie d'Argences, 1961, 253 p., seconde partie, chap. xvi : « Des trois choses principales que doit garder le Roi au fait de la Justice », p. 151.

2. Robert Bireley, The Counter-Reformation Prince. Anti-Machiavellianism or Catholic Statecraft in Early Modern Europe, Chapel Hill / Londres, The University of North Carolina Press, 1990.

3. Nomination par lettres patentes du duc de Berry du 16 mars 1582, confirmation par le roi du 28 mars de la même année, Archives départementales du Cher (AD Cher), C $964, f^{\circ} 26 \mathrm{v}^{\circ}-28 \mathrm{v}^{\circ}$; pour les dates des nominations de ses successeurs et les officiers royaux à Bourges en général voir Thomas Lüttenberg, Les Sens extérieurs de l'État. Königliche Amtsträger aus Justiz und Finanz in Bourges (1550-1650), thèse de $3^{\mathrm{e}}$ cycle, université de Bielefeld, à paraître.

4. Contrat de mariage entre Catherine Biet et Charles du Ménil-Simon du 21 février 1667, AD Cher, B 723, fo $79 \mathrm{r}^{\circ}-81 \mathrm{r}^{\circ}$.

5. Extraict et Inventaire de la preuve littérale de Maistres Pierre Bidault et Gabriel Manceron, conseillers, magistrats au siège presidial de Bourges, ... dénonciateurs pour le corps dudict siège presidial, pour faire procès à Maistre Pierre Biet, lieutenant general audit Bourges (1614), s.l.n.d., 47 p.

6. Ibid., p. 37.

7. Ibid., p. 6.

8. Ibid., p. 41.

9. Le contrat de mariage du père de Pierre Biet avec Françoise Suzanne Gassot, sœur du lieutenant particulier au siège présidial et futur prévôt de Bourges, Gabriel Gassot, du 20 janvier 1553 a été perdu, voir néanmoins, Bibliothèque nationale de France (BnF), cabinet des titres, dossier bleu $95 / 2281$, fo 11 ; il en est de même du contrat de mariage du lieutenant général avec Catherine Doullé du 12 août 1582 (BnF, cabinet des titres, dossier bleu 95 / 2281, fo 11 et $\mathrm{AD}$ Cher, $2 \mathrm{~F} 251, \mathrm{n}^{\circ} 28$, généalogie Doullé) ; voir également le contrat de mariage de la sœur du lieutenant général, Jeanne Biet, avec Robert Garnier, conseiller au présidial (AD Cher, E 4752, $\mathrm{n}^{\circ} 272,22$ novembre 1583) ; et les contrats de ses deux filles, Jeanne et Catherine, avec respectivement Claude Pellet, prévôt de la maréchaussée (AD Cher, E 4773, n 50, 26 février 1602) et Robert Heurtault, conseiller au présidial (AD Cher, E 2422, nº 278, 12 novembre 1609). 
10. Nomination de son fils aîné, Claude Biet, par lettres patentes du 10 août 1624, AD Cher, C $1001, \mathrm{f}^{\circ} 30 \mathrm{v}^{\mathrm{o}}-31 \mathrm{r}^{\mathrm{o}}$.

11. Extraict et Inventaire de la preuve littérale..., op. cit., p. 47.

12. Wolfgang Reinhard, éd., Die katholische Konfessionalisierung, wissenschaftliches Symposion der Gesellschaft zur Herausgabe des Corpus Catholicorum und des Vereins für Reformationsgeschichte (1993), Münster, Aschendorff, 1995; Michael Stolleis, «Grundzüge der Beamtenethik (1550-1650) », Roman Schnur, Die Rolle der Juristen bei der Entstehung des modernen Staates, Berlin, Duncker \& Humblot, 1986, p. 273-302, prône, par contre, au niveau européen, un rôle décroissant des confessions pour l'« éthique des fonctionnaires » entre 1550 et 1650.

13. Colin Kaiser, «Les Cours souveraines au XVI ${ }^{\mathrm{e}}$ siècle : morale et Contre-Réforme ", Annales ESC, 37, 1982, p. 15-31.

14. Carole Delprat, «Magistrat idéal, magistrat ordinaire selon la Roche-Flavin : les écarts entre un idéal et des attitudes ", Jacques Poumarède et Jack Thomas, éd., Les Parlements de Province. Pouvoirs, justice et société du XV $v^{e}$ au XVIII siècle, Toulouse, Framespa, 1996, p. 707-719.

15. Carole Delprat, art. cit., p. 710.

16. Carole Delprat, art. cit., p. 709 , note 7.

17. Colin Kaiser, se référant aux séances mercuriales du parlement de Paris des années 1550 , art. cit., p. 21.

18. L'importance politique de l'image des magistrats souverains est soulignée par Elie Barnavi et Robert Descimon, La Sainte Ligue, le juge et la potence. L'assassinat du président Brisson (15 novembre 1591), Paris, Hachette, 1985, p. 131, 170.

19. Voir à titre d'exemple les discussions lors des états généraux de Blois (1576), visant une extension des mercuriales à tous les cours provinciaux (présidiaux, bailliages); Georges Picot, Histoire des États Généraux considérés au point de vue de leur influence sur le gouvernement de la France de 1355 à 1614, 4 vol., Paris, Hachette, 1872, t. II, p. 488-489. L'exemple d'une cour provinciale est traité par Jonathan Dewald, The Formation of a Provincial Nobility. The Magistrates of the Parlement of Rouen (1499-1610), Princeton, Princeton University Press, 1980, 231 p., chap. I «Professional Identity and Professional Attachments ».

20. Inventaire de la preuve littérale..., p. 47.

21. Exemples des années 1551, 1554 et 1559, cités par Colin Kaiser, art. cit., p. 20.

22. Cité par Carole Delprat, p. 712.

23. Le Miroir royal des Louys, ou instruction politique de ce que les trois ordres peuvent légitimement demander au roy en l'assemblée des Estats, Paris, 1614, cité par Hélène Duccini, « Discours et réalité sociale : le révélateur des pamphlets », Henry Méchoulan, éd., L'État baroque (1610-1652), Paris, J. Vrin, 1985, 504 p., p. 369-420, p. 377.

24. Colin Kaiser, art. cit., p. 20 ; Carole Delprat, art. cit., p. 712 ; Olivier Christin, La Paix de religion. L'autonomisation de la raison politique au XVI ${ }^{e}$ siècle, Paris, Seuil, 1997, 327 p., p. 37 ; Daniel Roche, La Culture des apparences. Une histoire du vêtement (xvii ${ }^{e}$-xviiie siècle), Paris, Fayard, 1989, 549 p., p. 54 ; Neithard Bulst, éd., Les Lois somptuaires en France I: ordonnances royales (XIII ${ }^{e}-\mathrm{XVIII}{ }^{e}$ siècle), édition critique, Stuttgart, Thorbecke, à paraître.

25. Carole Delprat, art. cit., p. 712.

26. Louis d'Orléans, Les Ouvertures des Parlements, auxquelles sont adjoustées cinq remonstrances autrefois faictes en iceluy, Paris, Des Rues, 1607, fo $257 \mathrm{v}^{\circ}$; dans le même sens vont André Duchesne, Les Antiquitez et recherches de la grandeur et majesté des roys de France..., Paris, J. Petit-Pas, 1609, p. 490 et Bernard de La Roche-Flavin, Treize Livres des parlements de France, ès quels est amplement traité de leur origine et institution, et des présidents, conseillers, gens du roi, greffiers, secrétaires, huissiers et autres officiers..., Bordeaux, S. Millanges, 1617, liv. X, chap. XxIV ; Mical H. Schneider, The French Magistracy (1560-1615), Ann Arbor, 1981, p. 273-274.

27. Marcel Rousselet, Histoire de la magistrature française des origines à nos jours, 2 vol., Paris, Plon, 1957 , t. I, p. 331-333. 
28. Thomas Sonnet, sieur de Courval, Les Satyres contre les abus et desordres de la France (1621), satyre quatriesme, Prosper Blanchemain, éd., Euvres poétiques de Courval Sonnet, 3 vol., Paris, Librairie des bibliophiles, 1876-1879, t. I, p. 91.

29. Antoine Furetière, Dictionaire universel, contenant generalement tous les mots françois tant vieux que modernes..., 3 vol., La Haye/Rotterdam, 1690, art. « habit ».

30. Thomas Sonnet, sieur de Courval, Satyre contre les charlatans et pseudomédecins empyriques, en laquelle sont amplement descouvertes les ruses et tromperies de tous thériacleurs, alchimistes, chimistes, paracelsistes, distillateurs, extracteurs de quintescences, fondeurs d'or potable, ministres de l'elixir et telle pernicieuse engeance d'imposteurs..., Paris, J. Millot, 1610.

31. À titre d'exemple Édouard Fournier, éd., Les Caquets de l'accouchée (1623), nouvelle édition, Paris, P. Jannet, 1855, p. 22-23, 36-37.

32. Thomas Sonnet, Contre la corruptelle, p. 88 ; Colin Kaiser, art. cit., p. 16.

33. Benoît Garnot, «Le Bon magistrat et les mauvais juges à la fin du XVII ${ }^{\mathrm{e}}$ siècle », id., éd., Juges, notaires et policiers délinquants, XIV ${ }^{e}-X X^{e}$ siècle, Dijon, Éditions universitaires de Dijon, 1997, 205 p., p. 17-25, p. 23.

34. Thomas Sonnet, Contre la corruptelle, p. 93-94.

35. Il faudrait néanmoins penser à Sigmund Freud, "Charakter und Analerotik ", Anna Freud, éd., Sigmund Freud, Gesammelte Werke, chronologisch geordnet, t. VII, Francfort-sur-le-Main, Fischer, 1969, p. 201-209; Norman Oliver Brown, Life Against Death, Middletown, Wesleyan University Press, 1959, chap. XV « Filthy Greed ».

36. Lester K. Little, «Pride Goes Before Avarice: Social Change and the Vices in Latin Christendom ", The American Historical Review, 76, 1971, p. 16-49, p. 38.

37. Thomas More, La Description de l'isle d'Utopie..., Paris, Charles L'Angelier, 1550, fo $54 \mathrm{r}^{\circ}$.

38. Rabelais, Pantagruel (1532), chap. v, Mireille Huchon, éd., Rabelais, Euvres complètes, Paris, Gallimard, « Bibliothèque de la Pléiade », 1994, p. 231.

39. Pasquil ou Plaincte sur la Reformation des Habits, s.l.n.d. (BnF, Ye 29601).

40. Edict du roy portant reglement des habits..., Paris, F. Morel et P. Mettayer, 1613 (BnF, Actes royaux, F 46925/8) ; Denis Richet, "La Polémique politique en France de 1612 à 1615 », Roger Chartier et Denis Richet, éd., Représentation et vouloir politiques autour des états généraux de 1614, Paris, Éditions de l'EHESS, 1982, 194 p., p. 151-194.

41. Les définitions des étoffes proviennent toutes du dictionnaire de Furetière ; Maurice Leloir, Dictionnaire du costume et de ses accessoires, des armes et des étoffes des origines à nos jours, Paris, Gründ, 1951, 435 p.

42. Pasquil, p. 4-5.

43. Ibid., p. 5-6. 